\title{
RADIOISOTOPE CONFERENCE AT OXFORD
}

$\mathrm{T}$

HE Second Radioisotope Conference organized by the Atomic Energy Research Establishment, Harwell, was held at Oxford during July 19-23, with the object of discussing the experimental uses of radioisotopes and new techniques in their various applications in medicine, biology, agriculture, chemistry, physics, engineering and industry. The organizers of the Conference took the bold step of accepting only seventy-one out of more than two hundred. papers which were proffered; this removed the usual scourge of conferences-an overcrowded programme and multiple sessions in parallel. Another notable achievement was the issue of preprints of all the papers well in advance of the Conference, and the limitation of the time of reading each paper to ten minutes; this allowed a considerable amount of time for discussion, which was very lively and stimulating.

The Conference started off with several papers dealing with the therapeutic applications of radioactive isotopes. Some of these are used as external applicators much in the same way as radium, but utilizing the special advantages offered by the new radioactive substances. R. C. Tudway, H. F. Freundlich and T. S. M. Marshall (Bristol) used iridium-192 as a cervix applicator ; the soft $\gamma$-rays from iridium make shielding of neighbouring organs much easier. Similarly, yttrium-90, which was used by F. Crainz (Catania), lends itself to intra-uterine application, as it is a pure $\beta$-ray emitter. F. Ellis and R. Oliver (Oxford) demonstrated the use of tantalum wire containing tantalum-182 as an applicator ; the great flexibility of these wires makes them less likely to obstruct blood vessels than the rigid radium needles.

The internal use of radioisotopes in therapy depends on the ability to concentrate the radioactivity in the organ to be irradiated. One such method, which may be used for the treatment of cancer of the lung, was developed by E. E. Pochin, G. B. Cook, R. M. Cunningham, A. Hollman, F. Hudswell and B. R. Payne (University College Hospital, London, and A.E.R.E., Harwell). Colloidal gold, containing gold-198, is precipitated on sugar charcoal particles which are too large $(30-43 \mu$ in diameter) to pass through the lung capillaries. If injected intravenously they will therefore be concentrated in the lungs, with only a very small fraction reaching the liver. This preparation can also be localized in any specified area of the lung by means of cardiac catherization. J. Van der Werff (Nijmegen) described a somewhat similar use of bismuth-206 for deposition in the reticulo-endothelial system.

In the field of diagnosis, radioiodine is still the chief isotope employed in a variety of new problems. Very interesting results were obtained by $\mathrm{H}$. G. Thode, C. H. Jaimet and S. Kirkwood (Hamilton, Ontario) on the uptake of iodine-131 by the salivary glands. It appears that a simple procedure, the comparison of the activity in a sample of saliva with that in the blood, provides a very sensitive method of discriminating between various types of thyroid disorders; the mechanism of the uptake in the salivary glands is not quite clear. The possibility of diagnosing carcinoma of the thyroid by means of a simple iodine-13I test was described by J. Rotblat and G. Owen (St. Bartholomew's Hospital, London), and the technique of outlining the thyroid and the location of tumours was improved by $\mathrm{H}$. C. Allen, jun., J. R. Risser and J. A. Greene (Houston, Texas), who employed for this purpose a crystal spectrometer with a double discriminator coupled to a scanning and recording device. A comparison of the behaviour in the body of iodine-131 and its analogue astatine-211 has been carried out by J. G. Hamilton, P. WallaceDurbin and M. W. Parrott (Berkeley, California); astatine, being an $\alpha$-emitter, offers many advantages over the $\beta$-emitting iodine isotopes, but Dr. Hamilton emphasized the dangers involved in handling this element. Most of the experiments were carried out on rats; but the few studies on humans indicated some promising applications. The use of serum albumin labelled with iodine-131 for the determination of cardiac output was described by N. Veall, J. D. Pearson, T. Hanley and A. E. Lowe (Guy's Hospital and Hammersmith, London), who used a scintillation counter placed over the region of the heart and connected to a ratemeter and chart recorder ; the method has still to prove its value, but the advantages it offers as compared with older methods of cardiac output measurements merits further study.

Iron-59 has been used in several investigations carried out in the Royal Cancer Hospital, London. E. M. Ledlie and C. Baxter described some clinical applications of this isotope in hæmatological investigations, particularly in the study of erythropoiesis bone marrow diseases. L. F. Lamerton, E. B. Harriss and E. H. Belcher carried out animal experiments on erythropoiesis and the effects of X-ray irradiation; they obtained very interesting results on protection achieved by shielding of the spleen. S. Rowlands, T. Freeman and P. C. Fleming (St. Mary's Hospital, London) developed a technique for making blood volume determinations in animals by the simultaneous use of two isotopes, phosphorus-32 and chromium-51. The latter isotope was also used by J. S. Robertson, R. Milne and S. H. Cohn (San Franciseo) for observing the distribution of blood platelets in rats.

A somewhat unique use of radioisotopes was presented in a paper by F. P. Bowden, J. B. P. Williamson and $P$. Laing (Cambridge) on the metallic transfer in screwing and bolting which they studied by the autoradiograph technique using radiochromium. The connexion of this work with medicine arises from the fact that in operations on fractured bones a certain amount of metallic transfer may occur, sufficient to form an electrolytic couple which has a harmful effect on the healing of tissue.

St. Bartholomew's Hospital.

J. Rotblat

One session was devoted to methods of labelling compounds with carbon-14, tritium and iodine-131, three of the nuclides most used in medical and biochemical studies. In addition, the method of preparation of tritium used at the Atomic Energy Research Establishment was outlined by W. J. Arrol, 